\title{
Calculation of the normal section strength of rubcon beams using deformation diagrams
}

\author{
Aleksei Polikutin ${ }^{1, *}$, Yuri Potapov ${ }^{1}$, and Artem Levchenko ${ }^{1}$ \\ ${ }^{1}$ Voronezh State Technical University, 84, 20 let Oktyabrya st., 394006, Voronezh, Russia
}

\begin{abstract}
Rubber concrete (rubcon) - polymer concrete, which has high strength properties, as well as the constructions made of it. Fibrorubcon rubber concrete with the addition of disperse reinforcement. On the basis of test results for pure bending, empirical dependencies and prerequisites for adapting existing methods for calculating the strength of normal sections of rubcon and fibrorubcon beams were obtained. These studies allow developing methods for calculating the normal cross sections of bending elements made of rubber concrete using deformation diagrams and taking into account the work of stretched polymer concrete.
\end{abstract}

\section{Introduction}

Due to the large volume of production of synthetic rubbers in the Russian Federation, their use as a binder in the produce of polymer concrete is of practical interest due to the low cost compared to the already used polymer resins. In the works [1-4] was conducted multiple studies of the properties of this polymer concrete, on the basis of which the possibility of using this composite as a material for manufacturing structures for various purposes was proved.

A study of effective building structures engaged in a number of scientists, among which stands out the work S. Pinaev [5], and A. Polikutin [1-2], which is devoted to the study of bending elements made of polymer concrete. It is also worth noting that at the moment the main part of research is focused on epoxy and polyester polymer concrete and structures of them [6-13]. The development of calculation methods mainly applies to reinforced concrete bending elements [14-16] or reinforced concrete elements reinforced with FRP canvases [17]. At the same time, the methods of calculation of polymer concrete structures that exist today are mainly applicable to structures made of polyester, epoxy polymer concretes, due to the presence of a fairly wide base of empirical dependences [6]. These methods without special studies cannot be extended to structures made of rubber concrete.

\section{Strength calculations of rubcon beams}

As noted in [2], the strength of normal sections of bending elements from rubcon is primarily affected by the percentage of longitudinal reinforcement.

* Corresponding author: alevchenko@,vgasu.vrn.ru 
The analysis of the results of the experimental studies allowed us to establish the following prerequisites for calculating the strength of normal sections:

1. the calculation is carried out in stage III of the SSS;

2. the stress values in the rubcon of the compressed zone are determined by a three-line deformation diagram. The plot of compressive stresses has the form of a trapezoid;

3. part rubcon above the crack perceives tensile forces;

4. the stresses in the rebar, in case of destruction on a stretched area, are not more than the resistance to tension. The effect of fiber reinforcement (if any) on the strength of normal cross sections is taken into account by the equivalent area of rod reinforcement, perceiving similar tensile forces.

The distribution of the calculated internal forces and deformations in the section normal to the longitudinal axis of the bending element from rubcon in the 3rd stage of SSS, taking into account the above prerequisites, is shown in fig 1 .

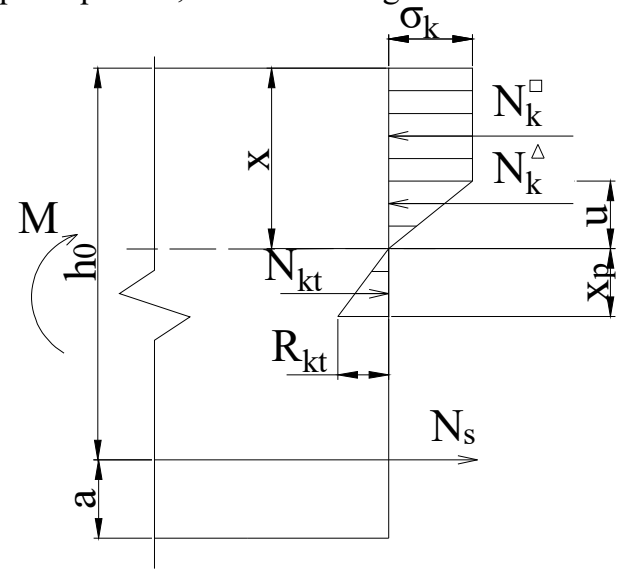

Fig. 1. Distribution of calculated internal forces in the normal sections rubcon bending elements in the calculation on the strength

The force in the rebar are determined by the formula:

$$
N s=R s=A s
$$

where Rs is the design resistance of the rebar. It should be noted that $\sigma \mathrm{t}$ - yield point was used instead of Rs in the calculation of the experimental beams.

$\mathrm{A}_{\mathrm{s}}$ - the area of rebar.

The force in rubcon stretched zones defined by the formula:

$$
N_{\mathrm{ht}}=\frac{1}{2} R_{\mathrm{ht}} \cdot x_{t} \cdot b
$$

where $x_{t}=\frac{\varepsilon_{\text {RE }}}{\varepsilon_{\mathrm{Z}}} x$;

$\varepsilon_{\text {htn }}$ - limit the extensibility of rubcon;

$\varepsilon_{\mathrm{n}}$ - compressive deformation of rubcon;

$\mathrm{b}-$ section width.

The force in the rubcon of the compressed zone is determined by the formula:

$$
N_{k}=N_{k}^{P}+N_{k}^{A}=a_{k}(x-u) b+\frac{1}{2} \sigma_{k}=u \cdot b \text {, }
$$

where $\sigma_{k}$ - stresses in the rubcon of the compressed zone determined on the basis of the three-line diagram of deformation shown in fig 2 obtained on the basis of testing of control samples - prisms $(4 \times 4 \times 16 \mathrm{~cm})$;

$u=x_{\hat{t}}$. 
The value of the height of the compressed zone is determined from the sum of the projections of internal forces on the longitudinal axis of the element:

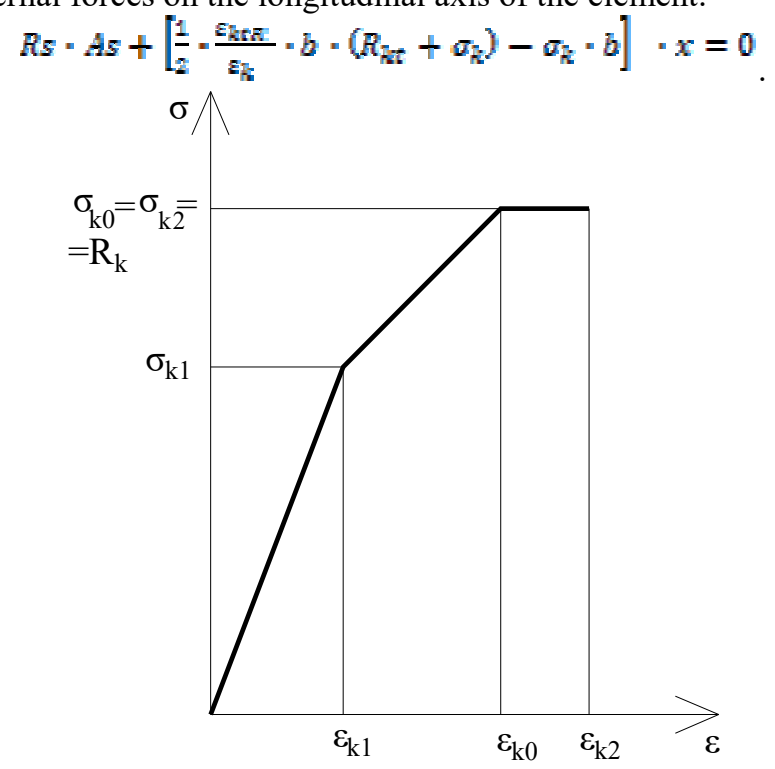

Fig. 2. Three-line stress-strain diagram of rubcon $\left(\sigma_{k 0}, \sigma_{k l}, \sigma_{k 2}-\right.$ compressive stresses at different loading stages; $\varepsilon_{k 0}, \varepsilon_{k 1}, \varepsilon_{k 2}$ - relative compressive strains at different loading stages)

The values of compressive stresses depending on the relative deformations are determined by the formulas:

$$
\begin{aligned}
& \text { When } 0 \leq \varepsilon_{\mathrm{k}} \leq \varepsilon_{\mathrm{k} 1} \\
& \sigma_{\mathrm{k}}=\mathrm{E}_{\mathrm{k}} \cdot \varepsilon_{\mathrm{k}} \cdot(5)
\end{aligned}
$$

When $\varepsilon_{\mathrm{k} 1}<\varepsilon_{\mathrm{k}}<\varepsilon_{\mathrm{k} 0}$

When $\varepsilon_{\mathrm{k} 0} \leq \varepsilon_{\mathrm{k}} \leq \varepsilon_{\mathrm{k} 2}$

$$
\sigma_{\mathrm{k}}=\sigma_{\mathrm{k} 1}+\left[\frac{\left(z_{\mathrm{k}}-z_{\mathrm{ki}}\right)}{\left(\mathrm{z}_{\mathrm{ke}}-z_{\mathrm{k} 1}\right)}\right]\left(\mathrm{R}_{\mathrm{k}}-\sigma_{\mathrm{k} 1}\right) .
$$

where $\sigma_{\mathrm{k} 1}=0.69 \mathrm{R}_{\mathrm{k}} ; \varepsilon_{\mathrm{k} 0}=0,0046 ; \varepsilon_{\mathrm{k} 2}=0,0055$;

$\mathrm{E}_{\mathrm{k}}$ - initial modulus of elasticity;

$\varepsilon_{\mathrm{k}}-$ the relative compressive deformation of rubcon;

$\mathrm{R}_{\mathrm{k}}$ - compressive strength of rubcon.

The bearing capacity is determined from the condition of equality of moments of all internal forces relative to the center of gravity of the stretched reinforcement:

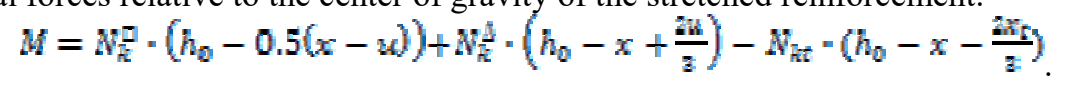

The results of calculation according to the given method in comparison with experimental values are given in Table 1.

For rubcon beams is allowed to determine the value of relative deformations depending on the percentage of longitudinal reinforcement according to the following empirical formula:

$$
\varepsilon_{\mathrm{k}}=(\mu+0,5) \cdot 10^{-3}
$$

The comparison of experimental and calculated values of the bearing capacity rubcon bending elements are shown graphically in fig 3 . 
Table 1. The calculated and experimental values of strength of normal sections rubcon bending elements using three-line diagram of deformation

\begin{tabular}{|c|c|c|c|c|}
\hline Beam cipher & $\mu, \%$ & $M_{u}^{\text {exp }}, \mathrm{kNm}$ & $M_{u}^{t}, \mathrm{kNm}$ & $\Delta . M, \%$ \\
\hline BRR -8 & 0.8 & 2.96 & 2.63 & 11,1 \\
\hline BRR -10 & 1.3 & 4.28 & 4.01 & 6.4 \\
\hline BRR -12 & 1.8 & 5.8 & 5.66 & 2.4 \\
\hline BRR -2x10 & 2.5 & 7.82 & 7.66 & 2.0 \\
\hline BRR -2x12 & 3.55 & 10.3 & 10.48 & -1.7 \\
\hline BRR -2x14 & 4.95 & 14 & 13.62 & 2.7 \\
\hline BRR -2x16 & 6.3 & 15.32 & 16.36 & -6.8 \\
\hline
\end{tabular}

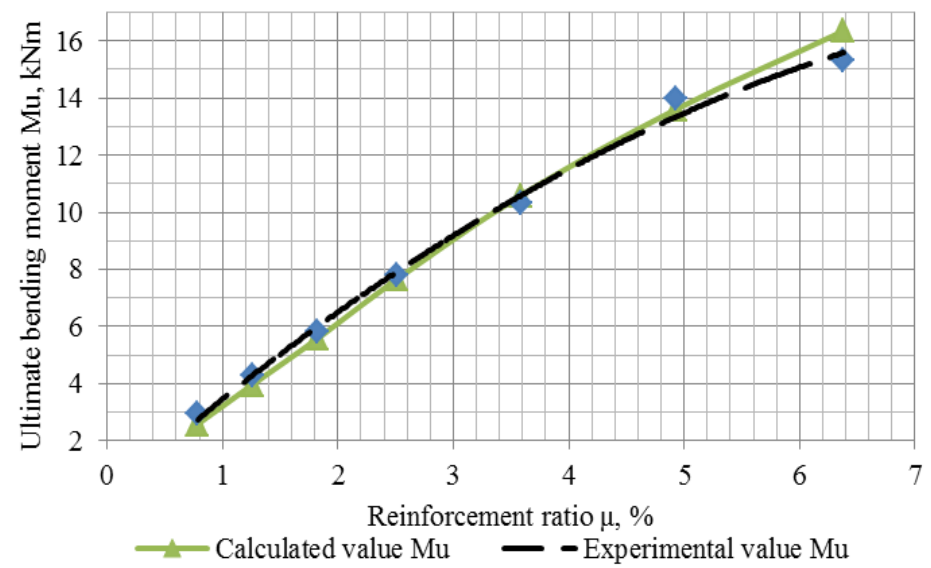

Fig. 3. Graphics of experimental and calculated strength of normal sections rubcon beams

As can be seen from fig 3 the curves corresponding to the experimental values and the calculated rubcon beams practically coincide.

\section{Strength calculations of fibrorubcon beams}

The distribution of the calculated internal forces and deformations in the section normal to the longitudinal axis of the bending element from fibrorubcon in the 3rd stage of SSS, taking into account the above prerequisites, is shown in fig 4.

The forces in the rebar are determined by the formula:

$$
N s=R s \cdot(A s+A f),
$$

On the basis of a series of tests of beams without longitudinal reinforcement [18], it was determined that an equivalent percentage of rod reinforcement, taking into account the work of the fibers of the steel cord in the normal section equal to $\sim 0.34 \%$. Consequently, for fibrorubcon bending elements, it is possible to take into account the effect of dispersed reinforcement in the calculation on the first group of limit states by adding a particulate rod equivalent area of reinforcement the area of longitudinal reinforcement. Value $A_{f}=0,2 \mathrm{~cm}^{2}$.

The force in fibrorubcon stretched zones defined by the formula:

where $x_{\mathrm{z}}=\frac{\varepsilon_{f \text { hr }}}{\varepsilon_{f k}} x$

$$
N_{f h t}=\frac{1}{2} R_{f h t}=x_{t} \cdot b \text {, }
$$

$\varepsilon_{f \text { f }}$ - limit the extensibility of fibrorubcon; 
$\varepsilon_{f k}$ - compressive deformation of fibrorubcon;

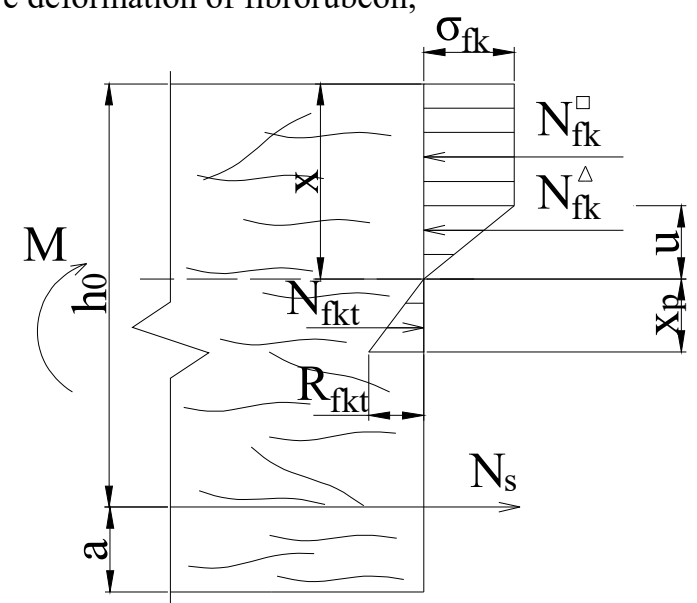

Fig. 4. Distribution of calculated internal forces in the normal sections fibrorubcon bending elements in the calculation on the strength

The force in the fibrorubcon of the compressed zone is determined by the formula:

$$
N_{f k}=N_{f k}+N_{f k}^{N}=\sigma_{f k}(x-u) b+\frac{1}{2} \sigma_{f k} \cdot u=b \text {, }
$$

where $\sigma_{\mathbb{R}}$ - stresses in the fibrorubcon of the compressed zone determined on the basis of the three-line diagram of deformation shown in fig 5 , obtained on the basis of testing of control samples - prisms $(4 \times 4 \times 16 \mathrm{~cm})$;

The value of the height of the compressed zone is determined from the sum of the projections of internal forces on the longitudinal axis of the element:

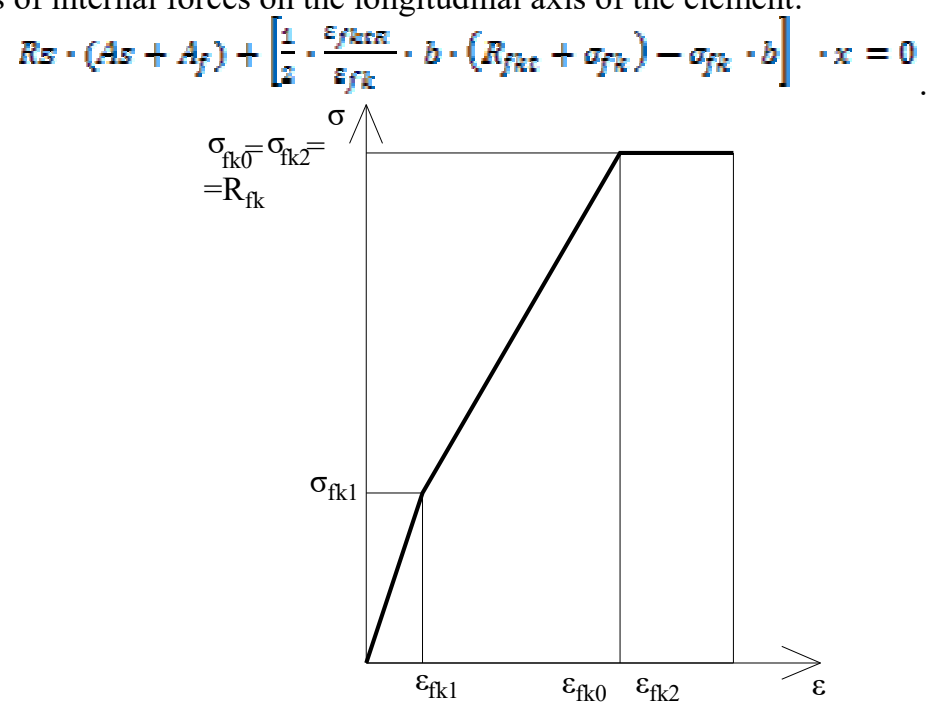

Fig. 5. Three-line stress-strain diagram of fibrorubcon

The values of compressive stresses depending on the relative deformations are determined by the formulas:

When $0 \leq \varepsilon_{\mathrm{fk}} \leq \varepsilon_{\mathrm{fk} 1}$

When $\varepsilon_{\mathrm{fk} 1}<\varepsilon_{\mathrm{fk}}<\varepsilon_{\mathrm{fk} 0}$

$$
\sigma_{\mathrm{fk}}=\mathrm{E}_{\mathrm{fk}} \cdot \varepsilon_{\mathrm{fk}} \cdot
$$


When $\varepsilon_{\mathrm{fk} 0} \leq \varepsilon_{\mathrm{fk}} \leq \varepsilon_{\mathrm{fk} 2}$

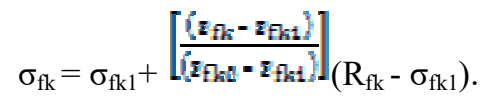

$$
\sigma_{\mathrm{fk}}=\mathrm{R}_{\mathrm{fk}}, \quad(16)
$$

where $\sigma_{\mathrm{fk} 1}=0.33 \mathrm{R}_{\mathrm{fk}} ; \varepsilon_{\mathrm{fk} 0}=0.0045 ; \varepsilon_{\mathrm{fk} 2}=0.0062$;

$\mathrm{E}_{\mathrm{fk}}$ - initial modulus of elasticity;

$\varepsilon_{\mathrm{fk}}-$ the relative compressive deformation of fibrorubcon;

$\mathrm{R}_{\mathrm{fk}}$ - compressive strength of rubcon.

The bearing capacity is determined from the condition of equality of moments of all internal forces relative to the center of gravity of the stretched reinforcement:

$$
M=N_{f}=\left(h_{0}-0.5(x-u)\right)+N_{f}=\left(h_{0}-x+\frac{z u}{g}\right)-N_{f h t}=\left(h_{0}-x-\frac{2 x_{5}}{g}\right) .
$$

The results of calculation according to the given method in comparison with experimental values are given in Table 2.

Table 2. The calculated and experimental values of strength of normal sections fibrorubcon bending elements using three-line diagram of deformation

\begin{tabular}{|l|l|l|l|l|}
\hline Beam cipher & $\mu^{\mu}, \%$ & $M_{u}^{\exp }, \mathrm{kNm}$ & $\begin{array}{c}M_{u}^{t}, \\
\mathrm{kNm}\end{array}$ & $\Delta . M, \%$ \\
\hline BRF - 8 & 0.80 & 3.78 & 3.68 & 2.6 \\
\hline BRF - 10 & 1.3 & 5.10 & 5.02 & 1.6 \\
\hline BRF - 12 & 1.8 & 6.77 & 6.61 & 2.4 \\
\hline BRF - 2x10 & 2.5 & 8.77 & 8.75 & 0.3 \\
\hline BRF - 2x12 & 3.55 & 11.28 & 11.73 & -3.9 \\
\hline BRF - 2x14 & 4.95 & 15.10 & 14.69 & 2.7 \\
\hline BRF - 2x16 & 6.3 & 17.52 & 17.55 & -0.2 \\
\hline
\end{tabular}

For fibrorubcon beams is allowed to determine the value of relative deformations depending on the percentage of longitudinal reinforcement according to the following empirical formula:

$$
\varepsilon_{\mathrm{fk}}=(\mu+1,1) \cdot 10^{-3}
$$

The comparison of experimental and calculated values of the bearing capacity fibrorubcon bending elements are shown graphically in fig 6

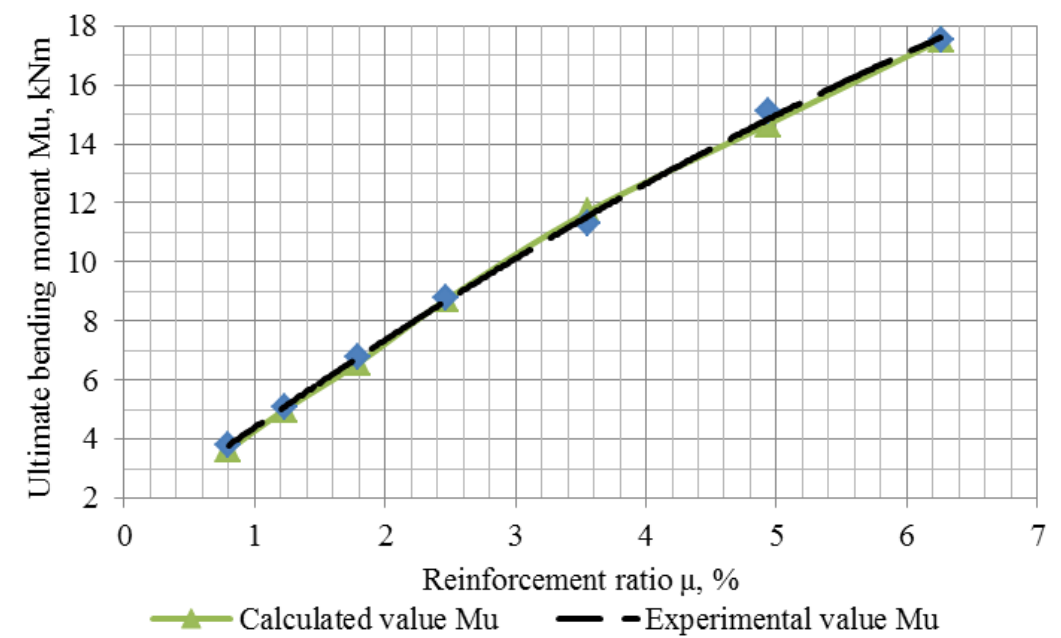

Fig. 6. Graphics of experimental and calculated strength of normal sections fibrorubcon beams 
As can be seen from fig 6, the curves corresponding to the experimental values and the calculated fibrorubcon beams practically coincide.

It should be noted that beams with a reinforcement percentage of more than 6.3 are not recommended for use due to their destruction in the compressed zone.

When calculating the ultimate bending moment using a three-line deformation diagram, the maximum deviation of the calculated bending moment from the experimental one is $11.1 \%$ in the BRR-8 series of beams, and $3.9 \%$ in the BRF- $2 \times 12$ series of beams.

\section{Conclusion}

To calculate the strength of the normal sections of rubcon bending elements destroying on the stretched zone ( $\mu=0.8$ to $6.3 \%$ ), it is recommended to apply the method of limit states taking into account the work of stretched polymer concrete, as well as using a three-line diagram of rubcon deformation.

To calculate the strength of the normal sections of fibrorubcon bending elements destroying on the stretched zone $(\mu=0.8$ to $6.3 \%)$, it is recommended to apply the method of limit states taking into account the work of stretched polymer concrete, as well as using a three-line diagram of fibrorubcon deformation. At the same time, to take into account the influence of dispersed reinforcement, it must be replaced by an equivalent area of rod reinforcement that perceives similar efforts.

\section{References}

1. A. E. Polikutin, IOP Conference Series: Materials Science and Engineering 463 (2018). doi:10.1088/1757-899X/463/2/022040.

2. Yu. Potapov, A. Polikutin, O. Perekal'skiy, A. Levchenko, Advances in Intelligent Systems and Computing 983 (2019). doi: 10.1007/978-3-030-19868-8.

3. O. Figovsky, Proceedings of the International Conference on Cement Combinations for Durable Concrete, 269-276 (2005).

4. O. Figovsky, D.Beilin, N. Blank Cement and Concrete Composites 18(6), 437-444 (1996).

5. Y.B. Potapov, S.A. Pinaev, A.A. Arakelyan, A.D. Barabash., Solid state phenomena, 871, 104-109 (2016).

6. H. Abdel-Fattah, M.M El-Hawary, Construction and Building Materials 13(5), 253-262 (1999).

7. J.M.L. Reis, Construction and Building Materials 20(9), 673-678 (2006).

8. R. Griffiths, A.Ball, Composites Science and Technology 60(14), 2747-2753 (2000).

9. W.O Oyawa, K. Sugiura, E. Watanabe, Construction and Building Materials 18(6), 367-376 (2004).

10. K.S. Rebeiz, S.P. Serhal, D.W. Fowler, Journal of Materials in Civil Engineering 6(1) (1994). https://doi.org/10.1061/(ASCE)0899-1561(1994)6:1(150)

11. M.J. Hashemi, M. Jamshidi, J.H. Aghdam, Construction and Building Materials 163, 767-775 (2018).

12. M. Hassani Niaki, A. Fereidoon, Structural Concrete 19(2), 366-373 (2018).

13. O.I. Németh, É. Lublóy, G. Farkas, Periodica Polytechnica Civil Engineering 58(2), 137-141 (2014). 
14. Jian-ren Zhang, Ke-bo Zhang, Hui Peng, Cheng Gui, China Journal of Highway and Transport, 2009-03.

15. Židonis Ipolitas, Procedia Engineering 57, 1309-1318 (2013).

16. Liu Sheng-bing, Li-hua Xu, Journal of Wuhan University of Technology 2012-11.

17. J.F. Liang, et al, Applied Mechanics and Materials 71, 815-817 (2011).

18. A.E. Polikutin, Yu.B. Potapov, A.V. Levchenko, Construction and architecture 1(22) (2019). doi 10.29039/article_5ca75fa 7e6c350.08575880. 